\title{
Development and Manufacture of the Coil End Spacers of the LHC Pre-Series Dipoles
}

\author{
E. Farina, P. Fessia, D. Perini, A. Schiappapietra, and L. Seneé
}

\begin{abstract}
The coil end spacers play an important role in the performance of superconducting coils, as their shape and location determine the mechanical stability of the conductors in the coil ends (and hence the overall coil training performance) and the local field quality.

The dipole end spacers are often of a size and a geometry difficult to be industrially series manufactured and measured. Efficiency of the production and related costs are a key issue to achieve the required production rate of the LHC main dipoles at an affordable price. For the latter reasons, a design approach integrating state-of-the-art CAD/CAM optimization techniques allowing to considerably decrease design and machining time was implemented. This paper gives examples and describes the design criteria, the computation methods, the machining and measuring procedures adopted to carry out the pre-series production.
\end{abstract}

Index Terms-Design of the surface, electromagnetic modeling, end spacers, machining.

\section{INTRODUCTION}

$\mathbf{T}$ HE PRODUCTION of the main dipole for the LHC is based on the mass production of many components; out of these components the end spacers are particularly challenging. The end spacers are the support of the superconducting cable at the coil ends. Here the conductor is bent as it exits the straight part of the coil, it climbs over the coil bore tube, and then comes back to the straight part on the opposite side. In this way the coil ends are saddle-shaped in order to allow beam in and out of the magnet. In addition to shaping the coil end, the end spacers have to guarantee the mechanical stability of the windings, too.

\section{RAW MATERIAL}

Usually the end spacers are made of epoxy-glass composite, such as G10 or G11. The aim of this work is to review both material and fabrication technique. The material should not become brittle in the severe working environment $(1.8 \mathrm{~K}$ and irradiation dose of 2 MGy in 25 years).

The use of two raw materials has been studied:

1) An engineering thermoplastic (production by molding).

2) An epoxy type thermoset (production by machining).

Manuscript received September 24, 2001. The work of A. Schiappapietra is supported by ASP (Associazione per lo Sviluppo Scientifico e Tecnologico del Piemonte).

E. Farina is with Tosti Srl, Castel del Piano (GR) I (e-mail: efarina@tostisrl.it).

P. Fessia, D. Perini, and A. Schiappapietra are with CERN, Geneva CH (e-mail: \{Paolo.Fessia; Diego.Perini; Alberto.Schiappapietra\}@cern.ch)

L. Senee is with Trimicron, Cluses, F.

Publisher Item Identifier S 1051-8223(02)04228-8.
TABLE I

RAW MATERIAL REQUiREMENTS ${ }^{1}$

\begin{tabular}{ll}
\hline Material property & Internal of acesptability \\
\hline Aval compressice strength & $>300 \mathrm{MPa}$ \\
Cohesion between layers & $>450 \mathrm{MPa}$ \\
Cohesion between logen afler 1 hour at $155^{\circ} \mathrm{C}$ & $>300 \mathrm{MPa}$ \\
\hline
\end{tabular}

A campaign of tests was run in order to study the feasibility of the use of molded end spacers. Different problems were discovered:

- Performance: to evaluate the introduction of molded pieces made of ULTEM ${ }^{\mathrm{TM}} 2300,{ }^{2}$ end spacers machined from extruded bars of the material were used to wind the coils of a short model. The performance results were not as good as expected showing that a more detailed and longer development effort was necessary.

- Production: due to the thickness of the pieces $(15.4 \mathrm{~mm})$ the injection process is extremely difficult to optimize. Bubbles, porous sections, cracks and poor tolerances are some of the problems put in evidence by the tests. To solve these problems a particular process using special molds has to be developed and tested, largely spoiling the economic advantage of the solution.

As results of the tests it was decided to continue to produce the massive pieces by machining, while the thin ones, chips and wedge tips, can be easily produced by injection molding. It has been proven that the introduction of these small components, made of ULTEM ${ }^{\mathrm{TM}} 2300$, does not deteriorate the performance of the magnets.

The raw material chosen for the production of the Main Dipole end spacer is designated as EP-GC-22 in the norm EN $61212-1$, as G11 in the norm NEMA LI-1 and as Hgw 2375.4 in the norm DIN 7735. The principle characteristics of this material are given in Table I.

This thermoset has been selected in order to stand the stresses induced in the spacers during the different phases:

1) Polymerization at $180^{\circ} \mathrm{C}$ of the coils under azimuthal load

2) Azimuthal pre-compression induced during assembly in order to withstand the electromagnetic forces

3) Axial electromagnetic forces arising during magnet excitation.

The experience highlights the risk of azimuthal cracks between two adjacent layers of the glass tissue used to wind the tubes. During the polymerization of the coils, this kind of defect can give rise to fracture of the pieces [Fig. 1(b)]. One of the causes

\footnotetext{
${ }^{2}$ Ultem is a trademark of General Electric.
} 


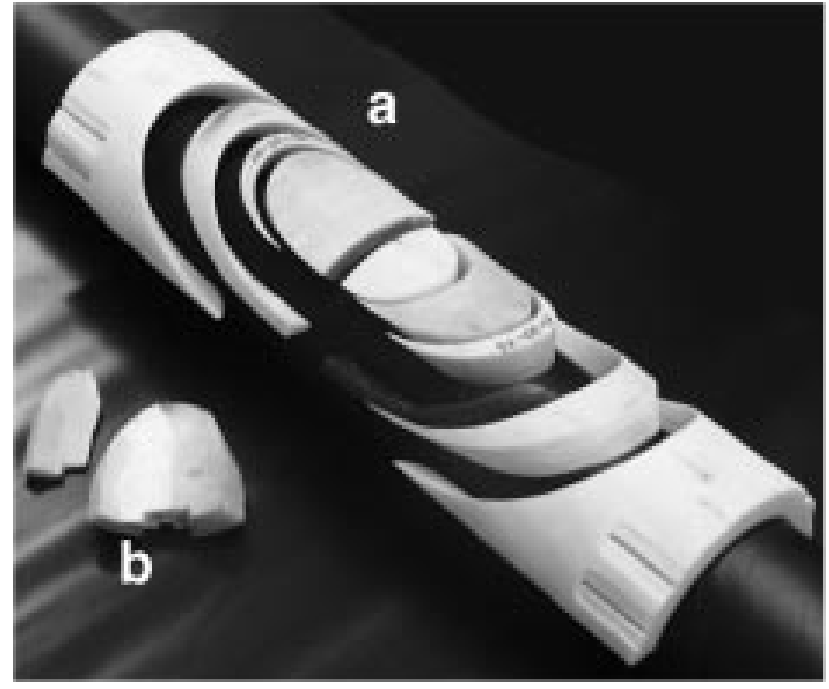

Fig. 1. (a) A complete set of outer layer end spacers and (b) one delaminated spacer.

of local weaknesses of the material, is the difficulty to guarantee, during the production of the tubes, the correct and uniform polymerization through the considerable thickness of the wall $(17-18 \mathrm{~mm})$. On the other hand the high values of axial compression strength are mandatory in order to withstand the axial component of the electromagnetic forces. Unfortunately the chemical parameters and the production processes required to improve the axial compressive strength, are detrimental to the cohesion between the layers and therefore a compromise has to be made.

\section{DESIGN PROCESS}

The choice of machining the end spacer for the LHC Main Dipoles defines also the way in which it is necessary to work in order to reduce the costs and guarantee the quality of the end spacers over the years necessary for the completion of the LHC dipole production. The work was done in the following phases:

- Design of the surfaces

- Tolerancing requirements

- Process of the surfaces in a CAD/CAM system and transfer of the surfaces to the end spacer manufacturer

- Machining

- Control.

\section{A. Design of the Surface}

For this series the surfaces were defined using dedicated software prepared at CERN. This software generates an end spacer starting from position of the conductors in the straight part of the magnet and from some basic geometric parameter as the thickness of the end spacer, or the winding angle. With these data the software determines the other parameters in order to get the same perimeter length between the upper and the lower edge of the end spacer. Each curve is made of an ellipse plus a straight part. The software is written in Mathematica ${ }^{\mathrm{TM}}$ in order to take advantage of its high precision and of its algorithms to solve elliptic integrals.
The shape of the end spacers is determined by applying the constant perimeter condition [1]. Applying such criteria in a more or less stringent way originates three different kinds of surfaces. In the first approach the upper and lower curves are divided into pieces with the same length. The equally spaced points are joined in the space with a straight segment. In the second one the height of the spacer $(15.4 \mathrm{~mm})$ is divided into equal intervals. At each level a curve (made again of an ellipse plus a straight segment) is computed in order to have the same perimeter as the upper and lower edges. The solution of many elliptic integrals is required. This surface has the same perimeter at all the levels to better receive the cable during the winding. In the third kind the discretization starts from the point where the ellipse is tangent to the straight part. The two parts of the curve are divided into the same number of segments. Due to the construction the length of these segments change from level to level and from the straight region to the elliptic one. The points are connected with lines. This last surface only differs respect to the second by some hundredths of a millimeter, but it allows much faster and easier machining [2].

\section{B. Tolerancing Requirements}

The geometrical requirements are translated into tolerances to be achieved and kept during the production. The limits have been set taking in account experience and simulations. In particular the simulations were used to estimate the effect, on the pre-stress profile, of piling up pieces at the limit of the acceptable interval of tolerance.

The dimensional requirements have a direct impact on the costs. These costs arise from the production technique that is necessary to keep the dimensional consistency and also the quality procedure that have to be put in place to verify correctly the production. The tolerances have been set in order to allow somewhat larger imperfections on the elliptic surfaces $( \pm 0.2 \mathrm{~mm})$ than on the thickness of the extremities $( \pm 0.06 \mathrm{~mm})$ which have to match the copper wedges.

\section{Process of the Surfaces in a CAD/CAM System and Transfer of the Surfaces to the End Spacer Manufacturer}

To avoid any problem of consistency the end spacers are modeled starting from an ASCII file, which contains the coordinates of the points describing the surfaces. Such a file is considered as the reference geometry. The decision has been taken not to refer to any IGS or other graphical file in order to avoid including, in the nominal geometry, errors aroused from software manipulations or from the conversion to the different type of IGS. The same ASCII file is transferred to the end spacer manufacturer who reads it into his own CAM system. It is therefore under the manufacturer's responsibility to define the tool path to get to the correct dimension for the lowest cost.

\section{Machining}

In order to achieve consistent cost reduction a program of development was launched to identify the best strategy to produce the end spacers. The development has gone through three main phases: 


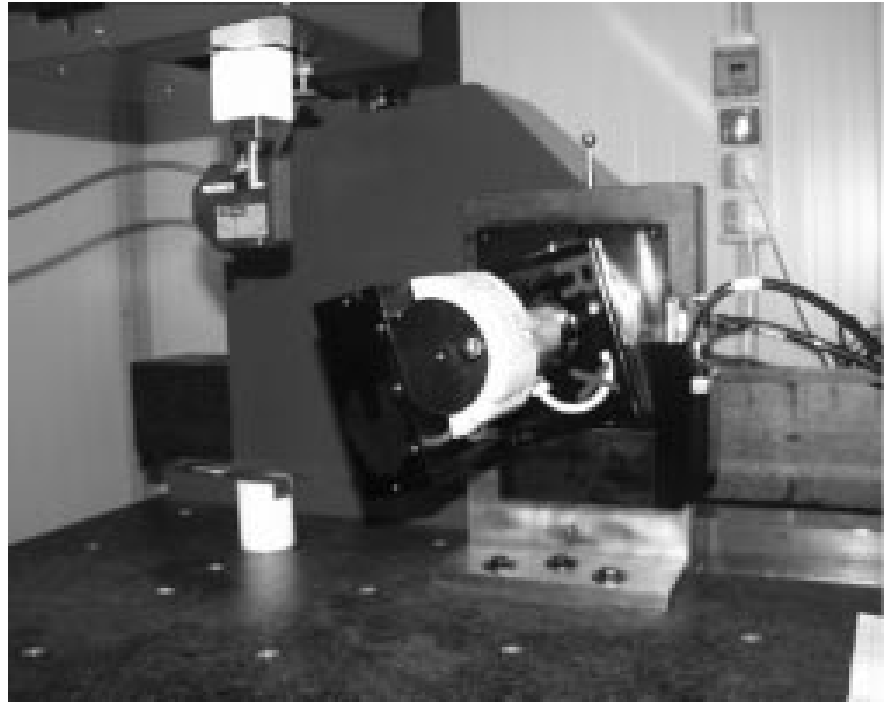

Fig. 2. The laser machine to measure end spacers.

1) Design for production and production cycle optimization: the surface topology has been tuned in order to provide the best interpretation by the CAM software and in order to allow 5 axis machining with a cylindrical cutting mill. The production cycle has been optimized in order to reduce costs and increase the process capability. Slight modifications of the surface description format have decreased the machining time and increased the quality.

2) Tooling optimization: the tooling to keep in place the pieces during the production has been designed to guarantee the best precision and to reduce the time needed to mount and demount the spacers.

3) The cutting mill head improvements: the cutting mills have been optimized as to the length, the design of the cutting edges and the surface. Longer tools provide more freedom to machine the pieces, but, due to their deflection, decrease the precision. The profile of the cutting edges and the surface coating have been optimized in function of the machining speed and of the quantity of material to be eliminated. An attempt has also been made to select tools that can stand longer use in contact with the abrasive glass fibers.

\section{E. Control}

The complexity of these components has required the development of an ad-hoc system to inspect them (Fig. 2). This tool makes use of a laser to acquire information on the piece that is fixed on a specially designed support. This support rotates in the space, exposing the whole object to the laser. Thanks to the system, the time to measure a piece can be reduced, increasing the quality and the quantity of the information collected. In addition the system can work at two different levels. In the simple inspection mode it checks the conformity of a selected number of key points, in the extensive analysis mode it surveys a large number of points (more than 100) creating a full map of the differences of the machined pieces with respect to nominal one. Another advantage of this approach is the possibility to get the information already coded in a software form

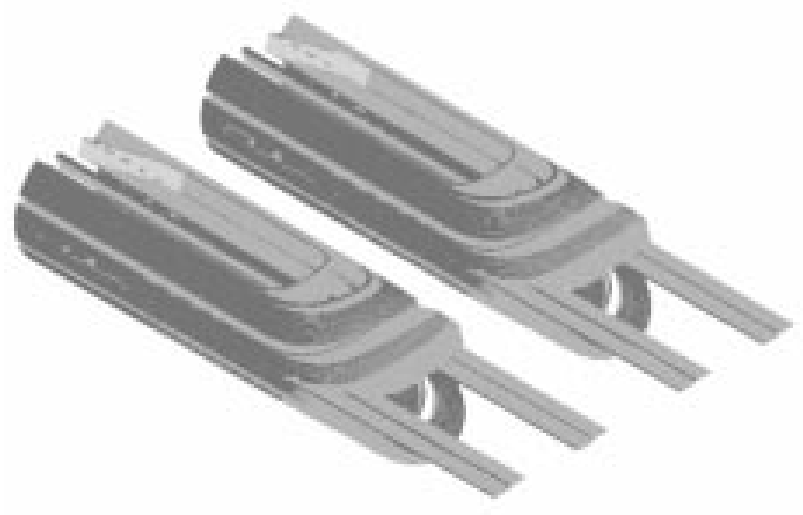

Fig. 3. Coil ends 3-D model realized by ROXIE, connection side.

and therefore ready to be integrated in the traveler and guaranteeing the traceability in time. As a consequence the handling of the data is faster and easier. From the point of view of the inspection plan, it has been decided to check all the pieces produced until it will be assessed that the process reliability is high enough to relax the control. After that, the inspection will proceed on a statistical basis.

\section{CHIPS AND WEDGE TIPS}

The chips and wedge tips are designed together with the main spacer, but the production is made by injection molding. The surfaces describing the pieces are transferred to industry where the molds are prepared by spark erosion. The molds are modular; this feature allows to change the shape of the pieces. The mold is made in such a way that the injection press produces a full set of pieces at each stroke. In the mold production, the shrinkage of the material has to be taken in account. Some tuning could be necessary and the production can be launched only after that the tooling has been accepted following the inspection of a trial run.

\section{Electromagnetic Modeling}

In order to validate the design, from an electromagnetic point of view, the geometrical parameters that define the end spacers are transferred to the numerical code ROXIE [3]. Thanks to this software the 3-D model (Fig. 3) of the heads is easily built and allows different checks and studies:

1) The verification of the peak field: the model allows computing the peak field in each cable of the winding.

With this information it is possible to perform appropriate changes to the geometry in order to reduce the value if it is not acceptable. In the example shown is the peak field in the first cable of the second layer before and after introducing an extra spacer, which separates the first and second turn from the third (Fig. 4).

2) The correlation between the effect of possible perturbations of the geometric parameters on the peak filed (Fig. 5) and on the harmonic content of the heads. This provides information to evaluate possible fine tuning of the field during the series production. 


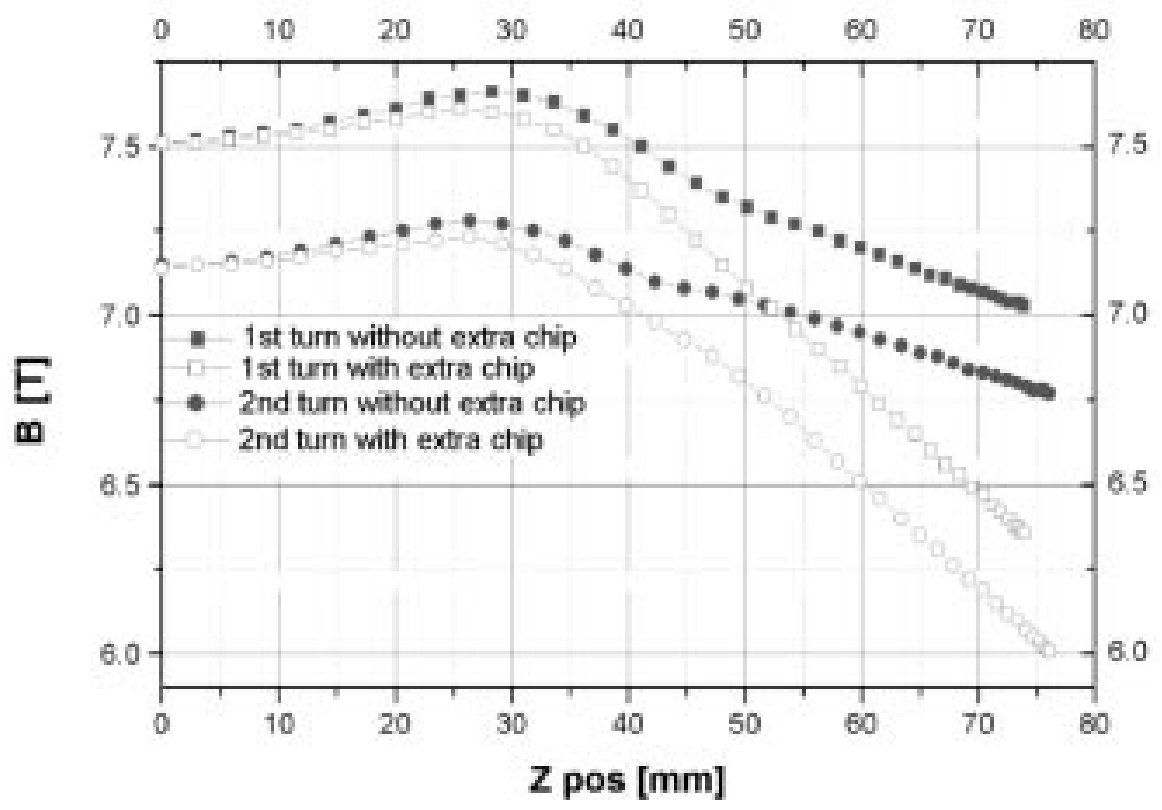

Fig. 4. The peak field in the first and second cable of the second layer with and without an extra chip.

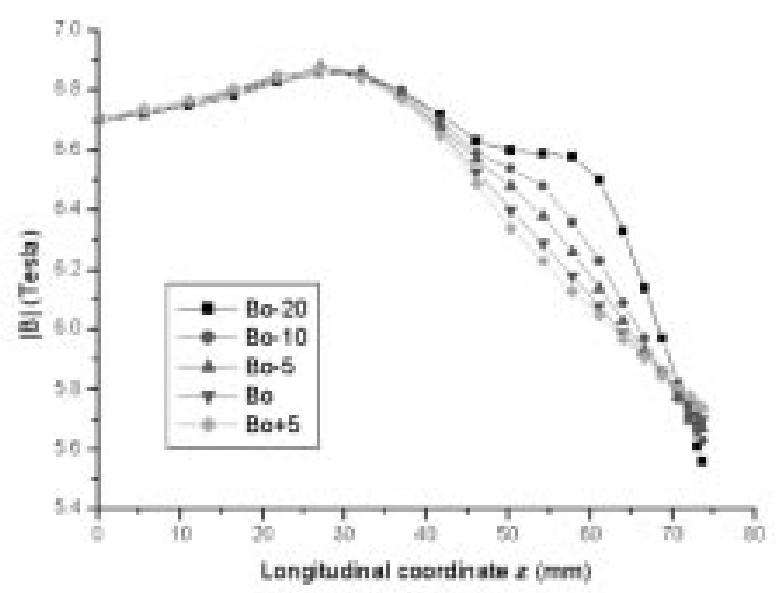

Fig. 5. Peak field variation in the very first cable of the outer layer due to the change of axis, $B o$, of the external surfaces of all the spacers (simulation realized by ROXIE).

It is shown that with the actual configuration large variations of the peak field on a cable or of the harmonic content can occur only as a consequence of large variations in the geometry. These variations are much larger then the ones that have been measured from coil to coil (Table II).

The understanding of the dimensional variation of the heads after curing has still to be improved, but the results available up to now show that the induced changes in the total harmonic inventory are negligible [4].

\section{CONCLUSION}

The design of the end spacers of the LHC pre-series main dipole has been approached, since the beginning, with the prime
TABLE II SENSITIVITY MAP

Sensitivity maps: the upper table shows the effect of the inclination angle of the spacer's surface over the multipoles b3 and b5; the lower table shows the effect of the spacer's longitudinal position over the multipoles b3 and b5 in coil end (simulation realized by ROXIE).

\begin{tabular}{|c|c|c|}
\hline $\max \operatorname{lng} a$ & $\begin{array}{c}\text { CAmLs INCuNation } \\
\text { mbipiodo }\end{array}$ & miaide \\
\hline \multirow[b]{2}{*}{ lind! } & b3 & 6.99 \\
\hline & bs & $\operatorname{ascos}$ \\
\hline \multirow{2}{*}{$\operatorname{biok} 2$} & b3 & 0.42 \\
\hline & Bs & 605 \\
\hline \multirow{2}{*}{ bhats } & bi & 02 \\
\hline & m & 01 \\
\hline \multirow{2}{*}{ bhat4 } & bj & was \\
\hline & b & asen \\
\hline & CABLES SHIFT & \\
\hline mar toyar & malipedo & anisinn \\
\hline \multirow{2}{*}{ bixk! ! } & b3 & 2 \\
\hline & bs & 6.97 \\
\hline \multirow{2}{*}{$\operatorname{sick} 2$} & b3 & 0.14 \\
\hline & b5 & as \\
\hline \multirow{2}{*}{ backls } & b) & 13 \\
\hline & ns & 0006 \\
\hline \multirow{2}{*}{ Hadt } & b] & 637 \\
\hline & 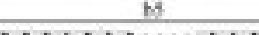 & 0.12 \\
\hline
\end{tabular}

objective of minimizing the production costs. To obtain such result all the phases, from the design, to the control, through the production have been optimized. The results are encouraging, showing that present and future suppliers would be able to deliver the requested product with high quality at limited costs. We are now working to improve the electromagnetic model of the heads to better predict the harmonic content of this section. The aim is to take into account all the mechanisms occurring during the winding, the polymerization and the magnet assembly in 
order to get to the most reliable model. This will be helpful to track effect of changes and drifts during the mass production of the main dipoles.

\section{ACKNOWLEDGMENT}

The authors would like to thank S. Cuzin for the help in the tests and S. Russenschuck for the fruitful discussions and help in establishing the ROXIE model.

\section{REFERENCES}

[1] G. H. Morgan and A. Greene et al., "Winding Mandrel design for the wide cable SSC dipole," Brookhaven National Laboratory, Upton, USA, Mar. 1991.

[2] W. L. Pope, M.A. Green, C. Peters, S. Caspi, and C. E. Taylor, "An alternate method for designing dipole magnet ends," Lawrence Berkeley Laboratory, University of California, Berkeley, CA, USA, Aug. 1988.

[3] S. Russenschuck, "ROXIE: Routine for the optimization of magnetic $\mathrm{X}$-sections, inverse field calculation and coil end design," in First Int. ROXIE Users Meeting and Workshop, CERN 16-18, March 1998, CERN 99-01.

[4] A. Schiappapietra, "Analisi elettromagnetica tridimensionale delle teste delle bobine nel dipolo principale di LHC," Tesi di Laurea, Politecnico di Torino, Dec. 2001, to be published. 